\title{
Author Correction: Democratic classification of free-format survey responses with a network-based framework
}

Tatsuro Kawamoto (D) and Takaaki Aoki (D)

Correction to: Nature Machine Intelligence https://doi.org/10.1038/s42256-019-0071-y, published online 9 July 2019.

In the version of this Article originally published, the $\sigma$ in '...the set of group assignments is denoted by $\sigma$ ' and equations (1) and (2) in the section 'Materials and methods' was incorrect; it should have been $\sigma_{i}$. Also, the sentence 'The degrees of vertices can be readily obtained from $A$ and $\sigma$ ' in the same section was incorrect; it should have read 'The degrees of vertices can be readily obtained from $A$ '.

Published online: 18 July 2019

https://doi.org/10.1038/s42256-019-0079-3 